\title{
La resiliencia: habilidad esencial para hacerle frente a la cuarta revolución industrial
}

\author{
Resilience: essential skill to face the fourth industrial revolution
}

\author{
Dr. Jesús Morgan Asch \\ Universidad Fidélitas, Costa Rica \\ jesus.morgan.asch@gmail.com
}

\section{RESUMEN:}

La cuarta revolución industrial se cierne sobre el mundo laboral como una amenaza significativa, al converger diversos avances tecnológicos disruptivos en el mismo espacio y tiempo de nuestra historia. Como todo cambio, esto representa un reto para la humanidad. El objetivo de esta revisión de literatura se centra en determinar cómo la resiliencia podría precisamente apoyar en el proceso de transición hacia la nueva revolución. Se determina que la resiliencia en sí misma es una filosofía que contiene muchas de las principales habilidades blandas que se requerirán en el futuro para hacer frente al cambio radical que se espera.

\section{ABSTRACT:}

The fourth industrial revolution looms over the world of work as a significant threat, while various disruptive technological advances are converging in the same space and time of our history. Like any change, this represents a challenge for humanity. The objective of this literature review focuses on determining how resilience can precisely support the transition process towards the new revolution. Resilience itself is determined to be a philosophy that contains many of the major soft skills that will be required in the future to face the expected radical change.

\section{RESUMO:}

A quarta revolução industrial paira sobre o mundo do trabalho como uma ameaça significativa, no momento que convergem vários avanços tecnológicos disruptivos no mesmo espaço e tempo da nossa história. Como qualquer mudança, isso representa uma adversidade para a humanidade. $\mathrm{O}$ objetivo desta revisão de literatura foi focado em determinar o modo como a resiliência poderia apoiar com precisão o processo de transição para a nova revolução. A resiliência em si mesma foi determinada como uma filosofia que contém muitas das principais habilidades sociais que serão requeridas no futuro para enfrentar as mudanças radicais esperadas.

\section{RÉSUMÉ:}

La quatrième révolution industrielle pèse sur le monde $\mathrm{du}$ travail comme une menace significative au moment où plusieurs avancées technologiques perturbatrices convergent dans le même espace et temps de notre histoire. Comme tout changement, cela représente un défi pour l'humanité. L'objectif de cette révision de littérature est centré sur la manière dont la résilience pourrait précisément favoriser le processus de transition vers la nouvelle révolution. Il est établi que la résilience en soi est une philosophie qui contient de nombreuses compétences générales importantes qui seront requises dans le futur pour faire face au changement radical attendu.

\section{PALABRAS CLAVE:}

RESILIENCIA, CUARTA REVOLUCIÓN, INDUSTRIAL, CAMBIO, HABILIDADES,
KEYWORDS:

RESILIENCE, FOURTH INDUSTRIAL, REVOLUTION, CHANGE, SKILLS.
PALAVRAS CHAVE:

RESILIÊNCIA,

QUARTA REVOLUÇÃO,

INDUSTRIAL,

MUDANÇA,

HABILIDADES,
MOTS CLÉS:

RESILIENCE, QUATRIEME REVOLUTION, INDUSTRIELLE, CHANGEMENT, COMPETENCES. 


\section{Introducción}

Esta investigación aborda el concepto de resiliencia y cómo puede ayudar a las personas y a las empresas para hacerles frente a los retos que se plantean con la cuarta revolución industrial.

La resiliencia se resume en resistir, aprender y trascender en medio de una adversidad. Sampedro (2009) define resiliencia personal como la capacidad de un individuo de afrontar adversidades, superarlas y salir transformado positivamente de ellas. Dentro de estas adversidades que enfrenta el ser humano, está presente el cambio. La cuarta revolución industrial viene a generar un cambio profundo, como se verá más adelante, en todos los aspectos de la vida como la conocemos. Schwab (2016) enmarca este concepto indicando que no se limita a tecnologías emergentes, sino hacia la transición a sistemas nuevos.

Como se verá en este artículo, las tendencias 4.0 son un conjunto de tecnologías que avanzan a una velocidad nunca antes vista en ninguna de las tres revoluciones industriales anteriores y con un impacto a nivel global sin precedentes, que se cierne sobre la humanidad como una gran amenaza. Esto, por cuanto se prevé que generará afectaciones en el empleo a gran escala y, si bien es cierto la tecnología ha ayudado a que ciertos puestos de trabajo desparezcan, no se habían proyectado escenarios como los que se analizan en la actualidad.

En la figura 1 se presenta el esquema conceptual de las repercusiones que tendrá esta revolución a todo nivel. Se puede observar cómo se proyectan mayores impactos en los siguientes ámbitos:

- empleos y habilidades

- gobernanza ágil

- innovación y productividad

- fusión de tecnologías

- disrupción empresarial

- ética e identidad

- desigualdad

- seguridad y conflicto

Figura 1: Impacto de la cuarta revolución industrial.

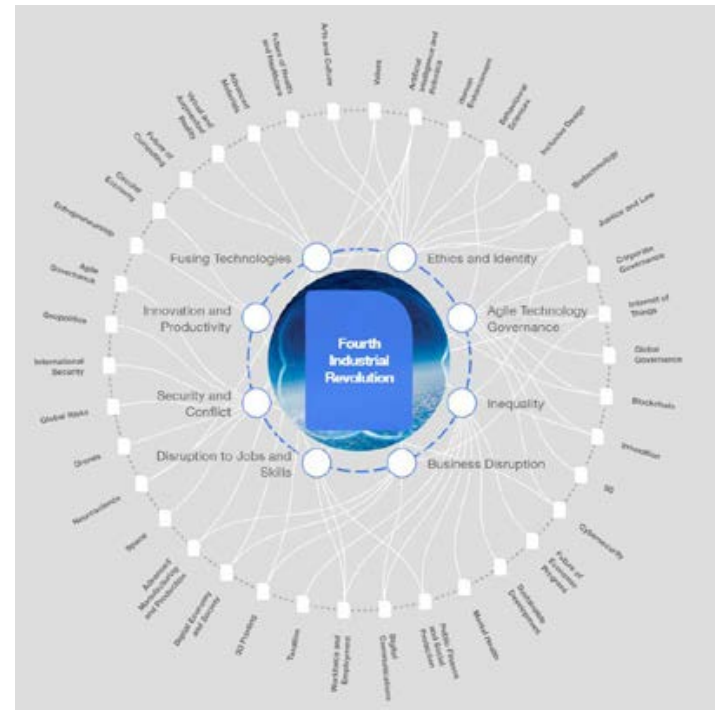

Fuente: Foro Económico Mundial (2020). 
Para el presente escrito, se efectuó una revisión documental, con el fin de responder a las siguientes interrogantes:

1. ¿Qué se entiende por resiliencia?

2. ¿Qué es la cuarta revolución del industrial?

3. ¿Cuáles habilidades se requieren para hacerle frente a la cuarta revolución industrial?

4. ¿Cómo contribuye la resiliencia al desarrollo de dichas habilidades?

Las tres revoluciones industriales anteriores generaron cambios en la sociedad, en los comportamientos humanos e incluso en la forma de hacer negocios. Según reseña Aurik (2017), la primera revolución industrial marcó el abandono de un modelo de producción artesanal doméstico y la migración hacia uno más estructurado y organizado en una instalación diseñada para esos fines. Esta revolución fue el primer reto que enfrentó la raza humana y se adaptó con una movilización hacias los centros urbanos desde los rurales, para encontrar trabajo. Además, se formaron las primeras organizaciones sindicales en defensa de los derechos de los trabajadores.

La segunda revolución industrial fue caracterizada por el uso de la electricidad en los procesos de manufactura. Potenció entonces la economía de escala y filosofías como el push, donde el paradigma que reinaba era ser lo más eficiente posible en los procesos de manufactura, sin saber si el mercado requería el producto. Ante la necesidad de movilizar grandes vólumenes de mercadería, el ser humano ideó nuevas redes de transporte y comunicaciones. Se adaptó incluso a través de la generación de nuevas profesiones como la ingeniería y la banca. A nivel social, surgió la llamada clase media, que buscaba cerrar brechas entre los más acaudalados y los más vulnerables, a través de políticas sociales solidarias.

La tercera revolución industrial trajo consigo las primeras vistas de la automatización en los procesos productivos. Particularmente, con el invento y la implementación de Internet, se potenciaron las comunicaciones y la globalización de mercados. Se generó una reconversión de empresas de manufactura a empresas de servicios y de la tecnología de la información. Cuando surgió el primer cajero automático, se pensó que sería el fin de los cajeros en los bancos y que la tecnología los reemplazaría por completo. Sin embargo, con una mentalidad de innovación y crecimiento, el ser humano los utilizó para potenciar más y mejores empleos en este sector, ejemplo último de cómo la tecnología podría colaborar con el crecimiento empresarial y de la calidad de vida tanto de los colaboradores como de sus clientes.

La cuarta revolución industrial se diferencia de sus predecesoras en tres puntos: velocidad, alcance e impacto en los sistemas (Schwab, 2016).

Como se puede observar, cada revolución ha implicado retos y transformaciones. El ser humano ha sabido optar por descubrir recursos con los cuales no contaba antes para hacerle frente a cada uno de los retos que se presentaron en su momento. Ahora, en la industria 4.0, existe un desfase, ya que la humanidad enfrenta retos de la cuarta revolución industrial con habilidades propias de la tercera. La tecnología y la invención crecen a niveles nunca antes vistos y de una forma cada vez más acelerada, que se proyecta como una amenaza y a la vez una oportunidad para diversos sectores en cuanto a pérdidas de empleo se refiere.

La resiliencia, como habilidad por antonomasia para enfrentar adversidades (en este caso, el cambio acelerado que implica la tecnología disruptiva), siempre ha existido. Su origen viene de la física, refiriéndose a la capacidad de un material para recuperar su forma original. La psicología la adaptó al ser humano, pero también se puede aplicar a una organización. La resiliencia organizacional se refiere al nivel de anticipación que posee una empresa para detectar tendencias que pueden ser favorables o desfavorables, adaptarse a ellas y recuperarse cuando se materialice alguna crisis(Dorantes, 2020). Lo anterior, sin perder la capacidad de cumplir su misión, sino, más bien, saliendo fortalecida e impulsada hacia el alcance de esta. Lo cual supone desarrollar habilidades que permitan que, de una manera innovadora, las empresas y sus colaboradores sorteen los diferentes retos que se presentan a su paso.

Los cambios tecnológicos generarán una transformación significativa en el ambiente laboral en los próximos años, producto de la cuarta revolución industrial. El ser humano, como principal activo de una organización, ya está enfrentando consecuencias en ese sentido. El desempleo es solamente una de ellas y no se puede evitar. Los empleos actuales no son los mismos que existirán en el futuro. La automatización, para nombrar solo uno de los tantos factores que se magnifican con esta revolución, podría generar una pérdida de empleos significativa en los diferentes sectores del mundo empresarial (Aurik, 2017). Sin embargo, no es la única consecuencia. Se ha indicado que se afectará el talento humano por causa de aspectos como el estrés, la ansiedad y el agotamiento. Es evidente, por lo tanto, que los empleadores deben emprender nuevos esfuerzos para cultivar lugares de trabajo positivos y saludables que mitiguen dichos impactos (AlMulla, 2018). 


\section{METODOLOGÍA}

La metodología utilizada fue una revisión de literatura, con el fin de identificar fuentes primarias y secundarias que aporten al conocimiento en relación con los temas de resiliencia y cuarta revolución industrial. La búsqueda comprendió la revisión documental de revistas, libros e investigaciones. Se utilizaron descriptores o palabras clave de búsqueda tales como "resiliencia", "cuarta revolución industrial", "cambio" o "habilidades". Además, se realizó una revisión en las principales bases de datos académicas: EBSCO, SciELO y Google Scholar. Este enfoque produjo un resultado de 46 referencias bibliográficas que van desde 1983 a 2018 (ver figura 2). De estas, solo 34 aportaron directamente al tema. Dentro de las principales limitantes del estudio, se puede mencionar que pocas fuentes bibliográficas enfocaban la resiliencia desde una perspectiva empresarial o laboral. El tipo de investigación es explicativa, bajo un paradigma hipotético deductivo, con un enfoque cualitativo.

Figura 2: Cantidad de publicaciones revisadas por año.

\section{Publicaciones analizadas por año}

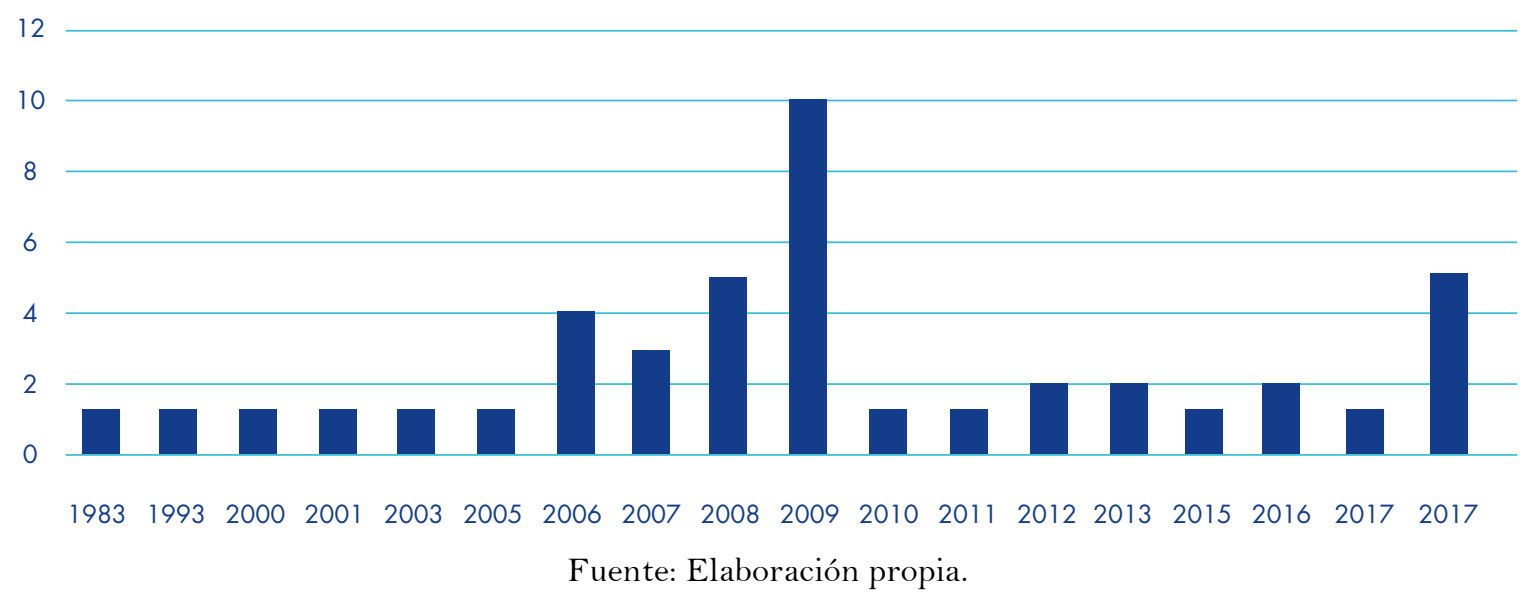

\section{Resiliencia}

La resiliencia forma parte de la psicología positiva. Esta es de carácter preventivo, más que correctivo. En síntesis, se concentra en generar en el individuo factores de protección para poder enfrentar situaciones difíciles, contrario al enfoque tradicional, donde la psicología viene a tratar de recuperar a una persona que haya caído en una crisis y tratar de curarla (Seligman y Csikszentmihalyi, 2000). Incluye también virtudes cívicas e institucionales que guían a los individuos a tomar responsabilidades sobre su comunidad y promueve características para ser un mejor ciudadano (Seligman y Csikszentmihalyi, 2000). También se define como el conjunto de recursos que tiene un individuo y que pueden incrementar su potencial (Sheldon y King, 2001).

La resiliencia es un concepto con un enorme potencial para todos aquellos profesionales que laboran en un determinado campo (Muñoz y De Pedro, 2005). Sus principios sencillos, pero poderosos, la hacen aplicable a cualquier rama o arista de la vida: económica, de la salud, social, personal, laboral y comunitaria, entre otras.

Se han encontrado correlaciones positivas entre la resiliencia y el compromiso del trabajador hacia la empresa, así como negativas, con respecto al agotamiento del trabajador, lo cual hace suponer que la resiliencia, en sí misma, puede proteger y fortalecer la fuerza laboral (Menezes de Lucena, Fernández Calvo, Hernández Martín, Ramos Campos y Contador Castillo, 2006). Las personas resilientes son capaces de experimentar emociones positivas en situaciones estresantes (Vecina, 2006).

La resiliencia organizacional se destaca como una cualidad deseada en las empresas e indispensable para el manejo de situaciones de crisis (Lalonde, 2007). Ruiz y Aguilar (2008) señalan como medidas para promover esta habilidad en los colaboradores el ser solidarios y generar un apoyo hacia los más débiles, por medio del trabajo en equipo, y crear 
una cultura donde todos confíen en que se puede ser exitoso, siempre dentro de límites realistas. Esto generaría un sentimiento de pertenencia hacia la empresa que fortalecería la cultura organizacional.

Bravo y Piñango (2008) establecen que, cuando una organización enfrenta una adversidad, sale fortalecida. Una razón es porque aprende de la experiencia; la otra es que la situación difícil generó un fuerte vínculo entre los colaboradores. Puntualizan además que las virtudes personales y organizacionales cultivan prácticas positivas, lo cual contribuye en la realización personal, el bienestar colectivo y la eficiencia. Esto genera un incremento en el desempeño organizacional. En este caso, se advierte que la resiliencia es parte del capital de la empresa y que genera un efecto de diferenciación sobre otras. Además, produce una mayor capacidad de adaptación a los cambios en su entorno.

Fiorentino (2008) retoma que las metas que se imponga una persona, empresa o país en esta materia deben ser realistas y alcanzables, para evitar niveles de frustración innecesarios. Esto genera una motivación real en los colaboradores al saber que, si se esfuerzan, perseveran y luchan, pueden verse recompensados en su propia vida.

La resiliencia a nivel social genera una mejora cualitativa en la calidad de vida, al concentrarse en las fortalezas personales. Una vez identificadas estas, se puede potenciar la calidad de vida y el desarrollo humano (Barranco, 2009).

Rodríguez Piaggio (2009) señala que el término resiliencia tuvo como origen la física, como una propiedad que determinaba la elasticidad de los materiales. La psicología adoptó esta propiedad y trasladó su uso a las personas, dando un significado positivo (visto desde la psicología positiva). Fue así como se utiliza el término para personas que, a pesar de las adversidades, pueden desarrollarse y ser felices. Esto, en situaciones donde hay alto riesgo de un desenlace negativo si no se enfrenta adecuadamente la adversidad.

Salanova (2009) también insiste en el concepto de que una organización que ha superado una adversidad se convierte en una organización resiliente, pues enfoca sus esfuerzos en generar una conciliación entre calidad de vida y desempeño laboral de sus colaboradores, a través de programas de salud y esfuerzos sistemáticos de mejora de ambas variables.

Para lograr este nivel de cultura, se deben facilitar espacios para la sensibilización y la capacitación, que motiven al colaborador a desarrollar habilidades de inteligencia emocional. El resultado de estos factores es tener una empresa más innovadora, productiva y finalmente más competitiva.

Una investigación realizada en la empresa fabricante de vehículos Hyundai obtuvo como resultado que la resiliencia, a pesar de ser poco común, es un aspecto esencial para el aprendizaje organizacional (Wright, Suh y Leggett, 2009).

La resiliencia puede desarrollarse como elemento preventivo para no caer en el agotamiento laboral (García Izquierdo, Ramos Villagrasa y García Izquierdo, 2009). Hay elementos a nivel individual que potencian la resiliencia, tales como la ecuanimidad para mantener la integridad frente a las dificultades y a las circunstancias cambiantes en el entorno, cambiar la mentalidad, aumentar la autoestima y redefinir la angustia como un elemento de protección y no de riesgo (Cesio, 2009).

En materia de gestión del talento humano, enseñar a los colaboradores a dominar sus propias emociones y que sean resilientes se vuelve un factor cada vez más determinante, incluso llevado a un nivel de política (Barrio y Barrio, 2009).

El propósito es que la persona se empodere y se adueñe de su propia existencia, y que a partir de allí pueda relacionarse mejor con otros de una manera reflexiva e incremente sus propios factores protectores y de resiliencia (Vilera, 2008). Dentro de los aspectos protectores clave para desarrollar la resiliencia, figura el tener expectativas realistas de lo que cada uno puede lograr, de acuerdo con sus propias capacidades. Esto impulsa a los colaboradores a comprometerse en mayor medida con sus actividades propias y mejorarlas, desarrollando, a su vez, sus propios talentos y habilidades, lo cual enriquecerá su abanico de posibilidades cuando surja alguna situación difícil que enfrentar (Fiorentino, 2008).

El ser humano, como un todo, es un ser integral. Tiene una dimensión física, una emocional y otra espiritual. Esta espiritualidad, sea religiosa o no, es una fuente poderosa que data de la antigüedad y, si es utilizada de la forma correcta, se puede convertir en un fuerte aliado para vencer cualquier adversidad (Naranjo, 2010).

Gómez (2012) propone que la resiliencia puede ser una medida eficaz para combatir la depresión y la ansiedad en forma preventiva. Profesionales de las ciencias de la salud y de las ciencias empresariales han discutido sobre los beneficios de brindar capacitación en el tema en empresas, escuelas y centros de salud, con el fin de aprender a superar una de las discapacidades más paralizantes que existen: el miedo. Si se tiene autoconfianza, flexibilidad, orientación al resultado, perseverancia y, ante todo, el sentimiento de querer enfrentar el problema, se tendrá resiliencia. 
Según Medina (2012), una persona resiliente no huye de la realidad; al contrario, la enfrenta. Además, en un ámbito laboral, presenta una mayor capacidad de resolución de problemas, en virtud de una mayor creatividad e innovación, para no enfocarse en las soluciones obvias, sino generar más alternativas poco usuales, pero efectivas, lo cual se convierte en una ventaja para las organizaciones. Este punto, recalcado por el autor, impacta en el mejoramiento continuo de los procesos de negocio, los cuales finalmente mejoran el desempeño de la empresa y la hacen más competitiva.

Medina (2012) habla de una simbiosis entre los colaboradores y una organización. Como resultado de esta interacción de doble vía, al incrementar los colaboradores su resiliencia personal, se incrementa el nivel de resiliencia de la organización y viceversa. A nivel macro, se habla de que uno de los factores externos que influyen en la resiliencia organizacional es el sector o industria al cual pertenezcan, así como el país o región de la cual sean miembros. En todos los niveles (personal, grupal, empresarial, sectorial y regional) existen retos y oportunidades para incrementar la resiliencia global.

Fiksel y otros (2015) incluso han abordado un enfoque específico sobre uno de los procesos empresariales más importantes, como lo es la administración de la cadena de suministro, y lo ligaron con el concepto de resiliencia. Su modelo ayuda a detectar las vulnerabilidades y posibles causas de riesgo que pueden materializarse y generar una interrupción en dicha cadena. Al contrastarla con la capacidad instalada, se identifican oportunidades de mejora y planes de acción que al final fortalecen a toda la empresa.

\section{Cuarta revolución industrial}

Esta revolución no es un conjunto de tecnologías emergentes. Es el proceso mismo de transición hacia sistemas totalmente innovadores que son y serán diseñados y construidos sobre la infraestructura digital que se heredó de la revolución anterior (Schwab, 2016).

Indica Schwab (2016) que hay tres diferencias que presenta la cuarta revolución con respecto a sus antecesoras y que la convierten en algo nunca antes visto: la velocidad del cambio (sin precedentes en la historia de la humanidad), el alcance global de este y el impacto en los sistemas tal como hoy los conocemos.

Schwab señala además cómo, por un lado, esta revolución traerá pérdida de empleos, pero, por otro, generará millones de nuevas oportunidades, solo que diferentes y únicamente para los que posean las capacidades y formaciones adecuadas. Las industrias y disciplinas se unirán. Los biólogos deberán ser también programadores y estadísticos.

Con base en lo expuesto por Schwab (2016), el reto para el talento humano es desarrollar esas habilidades técnicas o, como se les conoce también, habilidades duras. Para las empresas, el desafío será ayudarles a realizar esa transición por medio de la capacitación continua. Para los gobiernos, establecer políticas públicas orientadas a la transformación del sistema educativo y de las carreras que se imparten. Para la sociedad como un todo, tomar decisiones con base en principios morales y éticos que procuren el bienestar de todos.

Sin embargo, las habilidades duras no bastan. Schwab (2016) define habilidades blandas que deberán ser desarrolladas como parte de la sabiduría colectiva para adaptar, moldear y aprovechar el potencial de disrupción. El autor las encasilla en cuatro tipos de inteligencias:

- Contextual (la mente): cómo se entiende y aplica el conocimiento. Las personas líderes no pueden permitirse pensar en contextos reducidos. Su acercamiento a los problemas, las cuestiones y los retos debe ser integral, flexible y adaptativo, e integrar continuamente muchos y diversos intereses y opiniones.

- Emocional (el corazón): cómo se procesa y se integran los pensamientos y sentimientos, y cómo se relacionan con el individuo y con los demás. Esta inteligencia se hace aún más imprescindible en la era de la cuarta revolución industrial. Permite a las personas que ejerzan el liderazgo ser más innovadoras y agentes de cambio más efectivos. Las dota con habilidades tales como autoconocimiento, autorregulación, motivación, empatía y destrezas sociales. Se ahonda en este punto e inclusive se le da un mayor énfasis al indicar que, en un mundo caracterizado por el cambio persistente e intenso, las instituciones ricas en líderes con una alta inteligencia emocional no solo serán más creativas, sino que también estarán mejor equipadas para ser más ágiles y flexibles, un rasgo esencial para hacerle frente a la disrupción. La mentalidad digital, capaz de institucionalizar la colaboración multidisciplinaria, allanar las jerarquías y formar ambientes que fomenten la generación de nuevas ideas, depende profundamente de la inteligencia emocional. 
- Inspirada (el alma): cómo se utiliza el sentido de propósito individual y compartido, así como la confianza y otras virtudes para alcanzar un cambio y actuar en pro del bien común. El término alude a la continua búsqueda de significado y propósito. Se concentra en alimentar el impulso creativo y elevar a la humanidad a una nueva consciencia colectiva y moral, basada en un sentimiento compartido de destino.

- Física (el cuerpo): cómo se conservan la salud y el bienestar, con el fin de tener la energía suficiente para aplicar las transformaciones necesarias. Esto es fundamental porque, a medida que se acelera el ritmo del cambio, aumenta la complejidad y se incrementa el número de actores envueltos en los procesos de toma de decisiones. La necesidad de mantenerse en forma y conservar la calma bajo presión se torna aún más esencial.

\section{Habilidades para la nueva revolución industrial}

Se considera que, para 2020, un $35 \%$ de las habilidades clave en el mundo laboral actual habrán cambiado (Gray, 2016). Esto por cuanto las tecnologías disruptivas cambiarán las formas de hacer las cosas, tanto en el entorno laboral como en el empresarial, al aplicar y converger tecnologías como la automatización, la robótica, la impresión 3D, Internet de las cosas, el blockchain, la inteligencia artificial, la nanotecnología y otras.

En la figura 3, se presenta la evolución de las habilidades requeridas hace cinco años y lo que se demanda en la actualidad:

Figura 3. Habilidades requeridas en 2015 y 2020.

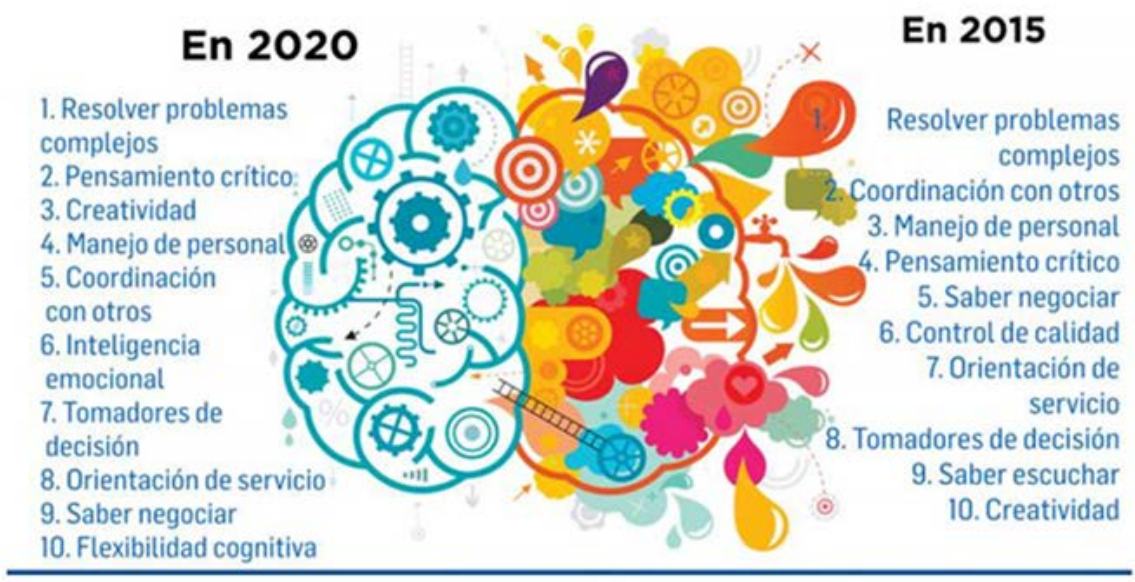

Fuente: Gray (2016).

En este apartado, llama la atención que habilidades que antes no eran clave, como la inteligencia emocional y la creatividad, sí lo son para el presente y para el futuro. Otras, por su parte, desaparecerían por ser repetitivas y de baja complejidad. Este análisis se ve reforzado con lo que expone Ordoñez Chillarón (2017), en lo que respecta a la inteligencia emocional. El impacto de la robótica, por ejemplo, se verá reflejado en oficios técnicos, principalmente. Sin embargo, se proyecta que, en combinación con otras tecnologías, el nivel de complejidad de las tareas que podrían asumir las máquinas es cada vez mayor.

Es entonces cuando el mundo empresarial torna su mirada hacia las habilidades blandas. Estas pueden generar nuevas fuentes de empleo al transformar puestos de trabajo existentes o incluso crear puestos que aún ni siquiera orbitan en la imaginación del ser humano. Ordoñez Chillarón (2017) define un nuevo concepto de trabajo: el trabajo emocional. Lo describe como aquel que integra las habilidades técnicas de un trabajador con sus emociones y sentimientos, donde el colaborador puede generar una situación empática con el cliente y su entorno.

Considerado lo expuesto, es clave que los trabajadores se adapten rápidamente a los nuevos cambios. Deben adquirir un amplio conjunto de habilidades que los ayudarán a sobrevivir en un mercado laboral. Entre ellas, figuran como las más importantes la resolución de problemas, el pensamiento crítico y la creatividad, además del hábito de aprendizaje 
permanente (Smith, 2018).

\section{La resiliencia y su relación con las habilidades para hacer frente a la cuarta revolución industrial}

Sierbert (2007) aborda cómo la resiliencia se relaciona con la resolución de problemas y la facilita a través del pensamiento crítico y la creatividad, habilidades principales requeridas para el futuro y lidiar con la cuarta revolución industrial. El autor se enfoca en la persona y en cómo esta puede generar ventajas a partir de su propio inventario de recursos. Normalmente, no se tiene la consciencia de estos recursos hasta que, por alguna razón, es necesario utilizarlos para superar adversidades. Los recursos varían desde el aspecto de salud, la resolución de problemas, la conciencia interior, habilidades de alto nivel y el talento de descubrir, hasta lo que él llama hallazgos afortunados. A medida que se potencie cada uno de estos factores, se potenciará la resiliencia en forma global.

La resolución de problemas se alza como la habilidad más significativa y que potencia en mayor medida la resiliencia (Sierbert, 2007). Para ello, es preciso enfocarse en la solución de una forma inteligente, en vez de estar pensar y profundizar en el problema de forma constante e improductiva.

Un colaborador resiliente es dueño de sus emociones. Al afrontar una crisis, domina en gran medida sus reacciones y las encausa a la solución de la problemática, en lugar de desgastarse en una reacción emocional. Será mucho más eficiente en utilizar sus recursos, reconocer las opciones y generar alternativas efectivas de solución de una manera más eficaz que alguien cuyos temores y ansiedades (para mencionar algunas) le dominen y afecten su razonamiento.

Dicha habilidad de resolución de problemas se basa en un proceso de identificación, examen, definición, clarificación y acción sobre la problemática o adversidad que se esté enfrentando. Se trata de un abordaje integral y lógico de la problemática, el cual deja de lado emociones como la ansiedad, la ira, el miedo, la sensación de vulnerabilidad y el sentimiento de estar desamparado.

Sierbert (2007) enumera emociones positivas (conocidas como factores de protección), que son útiles para enfrentar la adversidad y que potencian la solución efectiva de problemas. El solo hecho de pasarla bien, aunque las circunstancias sean adversas, es una ventaja que tendrá un colaborador sobre otro. Reír y divertirse generan sentimientos de empoderamiento; pasar momentos entrañables con amigos refuerza la red de contactos y disminuye el posible sentimiento de estar solo ante una situación adversa. Todas estas emociones, por básicas que parezcan, aumentan el inventario de recursos de la persona e incluso refuerzan su sistema inmunológico. Al final, se tendrá un colaborador fortalecido y listo para enfrentar el ajetreo diario.

La relación entre la resiliencia y la organización fue formalizada al promulgarse la norma ISO 22316:2016. Esta describe una serie de principios y atributos para fomentar la resiliencia organizacional, a la cual define como "la habilidad de una organización para absorber y adaptarse a un ambiente cambiante para llevar a cabo sus objetivos y para sobrevivir y prosperar" (ISO, 2016, p. 4). Además, señala que las organizaciones más resilientes anticipan y responden a las amenazas y oportunidades generadas por el entorno cambiante de una manera más efectiva, por lo cual indica que mejorar la resiliencia puede ser una meta estratégica organizacional y es el resultado de una buena práctica de negocios y de una gestión eficaz de los riesgos.

Como principales atributos de una organización resiliente, en dicha norma se citan los siguientes (ISO, 2016, p. 4):

- visión compartida y claridad de propósito,

- comprender e influir en el contexto,

- liderazgo eficaz y empoderado,

- cultura que apoye la resiliencia organizacional,

- información y conocimiento compartidos,

- disponibilidad de recursos,

- desarrollo y coordinación de disciplinas de gestión,

- apoyar la mejora continua,

- habilidad para gestionar y anticipar el cambio. 
Por otra parte, para lidiar con el estrés que generan eventos adversos tales como retos profesionales, enfermedades, cambios de trabajo y reestructuración organizacional, la resiliencia es clave (Marston y Marston, 2018). En lo que se refiere a la reestructuración organizacional, se puede contemplar el rediseño en las organizaciones basada en tecnologías de la cuarta revolución industrial. Marston y Marston (2018) indican cuáles habilidades se deben desarrollar para hacer frente a estas circunstancias:

- Replantear cómo el concepto sobre el estrés puede ser beneficioso

Marston y Marston (2018) hacen referencia a un estudio de Harvard de 2013 en el cual los investigadores dijeron a los participantes que los signos fisiológicos del estrés los preparaban para sobrellevar los problemas. Como resultado, los participantes se volvieron menos ansiosos y más seguros en situaciones estresantes, al saber que su respuesta al estrés era útil. Según se pudo observar, sus corazones y vasos sanguíneos respondieron de la misma forma en que lo hubieran hecho en tiempos de intensa felicidad.

- Estrés

Según Marston y Marston (2018), el personal debe hacer una introspección para determinar cuáles son los factores internos o externos que estén generando estrés. Incluso, se debe buscar retroalimentación con amigos, familiares o compañeros de trabajo para verificar si su perspectiva sobre los elementos estresantes es válida y poder preguntarse cómo abordarlos de una manera diferente.

- Crear una relación sana con el control

Se debe separar lo que puede ser controlado y lo que no; hacer un análisis sobre los eventos adversos y determinar cuánta influencia se tiene sobre ellos. Así se pueden tomar acciones para adaptarse o replantear el enfoque.

- Entender la causa raíz

Reflejarse dentro del contexto personal y organizacional para entender el entorno e identificar posibles cursos de acción para aliviar la carga de estrés.

- Enlazar el aprendizaje con la acción

Aprender del estrés que se experimenta y analizar qué se puede mejorar. Sin embargo, Marston y Marston (2018) indican que el análisis por sí solo no basta; hace falta implementar acciones de mejora que empiecen por uno mismo. Una modalidad que se ha desarrollado ha sido el coaching para motivar a los colaboradores en tiempos de incertidumbre laboral. Se busca mejorar el trabajo en equipo y cultivar en los colaboradores de la empresa una mentalidad de crecimiento (Moraleda, 2018).

El emprendimiento, factor clave en estos tiempos de industria 4.0, toma como base la terquedad de la resiliencia, conocida también como perseverancia (Dorantes, 2018). Resiliencia no se trata de solo resistir y resistir; se trata de aprender y trascender por encima de las adversidades. Es un factor que todas las personas desarrollarán en mayor o menor grado desde la niñez hasta la edad adulta. Dorantes (2018) señala que uno de los principales factores de protección es la autoestima. El creer que se puede superar los retos con las propias capacidades es una alta fuente de resiliencia y una clave que incidirá en ese mayor o menor grado de desarrollo mencionado antes.

Todo emprendedor resiliente debe desarrollar el sentido de la anticipación de las crisis: situaciones tales como una caída abrupta en las ventas, la pérdida de información, robos, hurtos o desastres naturales que puedan afectar su línea de negocio o su proyecto. Una vez visualizada la situación adversa, se debe crear un plan de acción para mitigar las consecuencias y los posibles escenarios, sin perder de vista los objetivos.

\section{CONCLUSIONES}

Al revisar los dos grandes conceptos abordados en este artículo, se puede observar la relación entre la resiliencia y la adversidad. El cambio, que normalmente se percibe como una amenaza, hace necesario que se recurra a habilidades blandas, tales como la resiliencia, para hacerle frente.

- La resiliencia es la capacidad, a nivel personal u organizacional, de anticipar, resistir, recuperarse y evolucionar al enfrentar una adversidad. A través de la revisión de literatura, se evidencia cómo esta filosofía ayuda a transformar positivamente al personal y a las empresas.

- Lo anterior se vuelve más significativo cuando la cuarta revolución industrial, definida como el proceso 
mismo de transición hacia sistemas totalmente innovadores que son y serán diseñados y construidos sobre la infraestructura digital, representa una amenaza para las empresas, los gobiernos y la sociedad. En el campo laboral, se observa que las proyecciones son negativas en cuanto a pérdidas de empleo e impacto económico; esto por cuanto generará cambios sustantivos a los que la humanidad deberá adaptarse.

- Al hacer un repaso por las tres revoluciones anteriores, la cuarta se diferencia en velocidad, impacto en los sistemas y alcance. Sin embargo, la historia muestra que la humanidad ha aplicado la resiliencia anteriormente; ha podido resistir el cambio y la adversidad, ha aprendido de esta, ha trascendido y evolucionado en cada una de las pruebas a las que se vio sometida.

- La resiliencia es esa virtud para adaptarse a los cambios, enfrentar adversidades y superarlas. Se constituye en una excelente herramienta para enfrentar cualquier crisis, ya sea a nivel personal o empresarial. Al analizar los resultados de la literatura investigada y comparar las habilidades blandas que se requerirán en el futuro, la resiliencia aporta fuertemente a muchas de ellas, tales como la resolución de problemas, el trabajo en equipo y otras.

- A pesar de que la resiliencia ha existido como propiedad física de los materiales y se ha utilizado en el área de la psicología, ha venido tomando un auge importante en el sector de las ciencias empresariales. Prueba de esto es la norma ISO 22316:2016. Dicha norma sigue esa dirección y se genera con el fin de fortalecer la gestión organizacional y la adaptación al cambio, precisamente los retos más importantes que presenta la cuarta revolución industrial. Pero, quizá lo más importante, y así lo refrenda dicha norma, es el desarrollo de la capacidad de adaptación al entorno cambiante, en tiempos en los cuales, por los efectos de la cuarta revolución industrial, el cambio será exponencial en todos los niveles.

Se recomienda, como una línea de investigación futura, profundizar cómo las habilidades resilientes pueden servir de diagnóstico para determinar el nivel de resiliencia en los colaboradores de las empresas, los gerentes y las organizaciones como un todo.

\section{REFERENCIAS}

AlMulla, A. (2018). “5 pasos para desarrollar resiliencia emocional en el trabajo”. Foro Económico Mundial. Recuperado de https://www.weforum.org/es/agenda/2018/02/5-pasos-para-desarrollar-resiliencia-emocional-en-el-trabajo

Aurik, J. (2017). “La cuarta revolución industrial tendrá un efecto disruptivo sobre el empleo, ¿pero cómo?” Foro Económico Mundial. Recuperado de https://www.weforum.org/es/agenda/2017/01/la-cuarta-revolucionindustrial-tendra-un-efecto-disruptivo-sobre-el-empleo-pero-como

Barranco, C. (2009). Trabajo social, calidad de vida y estrategias resilientes. Portularia, 9(2), 133-145.

Barrio, L. y Barrio, S. (2009). El factor emocional en auditoría (II). La gestión emocional en la evaluación del entorno de control. Auditoría Pública, 49, 75-85.

Bravo, O. y Piñango, R. (2008). Organizaciones que se atreven a desafinar. Debates IESA, 13(4), 89-92.

Cesio, S. (2009). El “burn out”: el síndrome de estar quemado en el trabajo. Revista Internacional de Psicología, 4(1).

Dorantes, R. (2018). Qué es la resiliencia y por qué la necesitas para ganar. Foro Económico Mundial. Recuperado de https://es.weforum.org/agenda/2018/o2/que-es-la-resiliencia-y-por-que-la-necesitas-para-ganar

Fiksel, J., Polyviou, M., Croxton, K. L. y Pettit, T. J. (2015). From Risk to Resilience: Learning to Deal With Disruption. Harvard Business Review. Recuperado de https://hbr.org/product/from-risk-to-resilience-learning-to-dealwith-disruption/SMR515-PDF-ENG

Fiorentino, M. T. (2008). La construcción de la resiliencia en el mejoramiento de la calidad de vida y salud. Suma Psicológica, 15(1), 95-114.

Foro Económico Mundial (2020). Cuarta Revolución Industrial. Recuperado de https://intelligence.weforum.org/ topics/a 1 Gboooooo 1RIhBEAW? tab=publications

García Izquierdo, A., Ramos Villagrasa, P. y García Izquierdo, M. (2009). Los Big Five y el efecto moderador de la 
resistencia en el agotamiento emocional. Revista de Psicología del Trabajo y de las Organizaciones, 25(2), 135-147.

Gómez, Á. (2012). . Manual de Resiliencia para no sucumbir ante la crisis. Expansión Recuperado de https://www. expansion.com/2012/03/02/entorno/1330718369.html Gray, A. (2016). The 10 skills you need to thrive in the Fourth Industrial Revolution. World Economic Forum. Recuperado de https://www.weforum.org/ agenda/2016/01/the-10-skills-you-need-to-thrive-in-the-fourth-industrial-revolution/

ISO (2016). Security and resilience. Organizational resilience. Principles and attributes. ISO.

Lalonde, C. (2007). The Potential Contribution of the Field of Organizational Development to Crisis Management. Journal of Contingencies \& Crisis Management, 15(2), 95-104.

Marston, A. y Marston, S. (2018). To Handle Increased Stress, Build Your Resilience. Harvard Business Review. Recuperado de https://hbr.org/2018/02/to-handle-increased-stress-build-your-resilience

Medina Salgado, C. (2012). La resiliencia y su empleo en las organizaciones. Gestión y Estrategia, 41, 29-39.

Menezes de Lucena, V. A., Fernández Calvo, B., Hernández Martín, L., Ramos Campos, F. y Contador Castillo, I. (2006). Resiliencia y el modelo Burnout-Engagement en cuidadores formales de ancianos. Psicothema, 18(4), 791-796.

Moraleda, A. (2018). Cómo motivar al trabajador en tiempos de inseguridad laboral. El País. Recuperado de https:// retina.elpais.com/retina/2018/02/06/talento/1517912850_105952.html

Muñoz, V. y De Pedro, F. (2005). Educar para la resiliencia. Un cambio de mirada en la prevención de situaciones de riesgo social. Revista Complutense de Educación, 16(1), 107-124.

Naranjo, R. (2010). Neurología de la resiliencia y desastres. Revista Cubana de Salud Pública, 36(3), 270-274.

Ordoñez Chillarón, E. (2017). La inteligencia emocional será la base del éxito laboral en el futuro. Yorokobu. Recuperado de http://www.yorokobu.es/inteligencia-emocional-y-trabajo-futuro/

Rodríguez Piaggio, A. M. (2009). Resiliencia. Revista Psicopedagogia, 26(80), 291-302.

Ruiz Guzmán, J. L. y Aguilar Valdés, A. (febrero-mayo, 2008). La inteligencia emocional como factor clave para la competitividad de las Pymes. Revista Virtual Universidad Católica del Norte, 23.

Salanova, M. (2009). Organizaciones saludables, organizaciones resilientes. Gestión práctica de riesgos laborales: integración y desarrollo de la gestión de la prevención, 58, 18-23.

Sampedro, J. A. (2009). Ingenio estrétegico. Resiliencia e impulso creativo en tiempos de crisis. Global Leadership Consulting. Recuperado de http://www.glcconsulting.com.ve/articulos/Articulo_Ingenio\%20Estrategico_ Jesus\%20Sampedro.pdf

Schwab, K. (2016). La cuarta revolución industrial. España: Penguin Random House.

Seligman, M. y Csikszentmihalyi, M. (2000). Positive Psychology: An introduction. American Psychologist, 55(1), 5-14.

Sheldon, K. y King, L. (2001). Why positive psychology is necessary. American Psychologist, 56, $216-217$.

Sierbert, A. (2007). La Resiliencia. Construir en la adversidad. Barcelona: Alienta Editorial.

Smith, R. (2018). ¿Para qué trabajos del futuro está mejor cualificado? Foro Económico Mundial. Recuperado de https://www.weforum.org/es/agenda/2018/02/para-que-trabajos-de-manana-esta-mas-cualificado/

Vecina, M. L. (2006). Emociones Positivas. Papeles del Psicólogo, 27(1), 9-17.

Vilera, A. (2008). Desarrollo humano y sentido de existencia: Abordajes desde un enfoque de orientación transformadora. Revista de Teoría y Didáctica de las Ciencias Sociales, 13, 29-52.

Wright, C.; Suh, C. S. y Leggett, C. (2009). If at first you don't succeed: globalized production and organizational learning at the Hyundai Motor Company. Asia Pacific Business Review, 1(2), 163-180. 